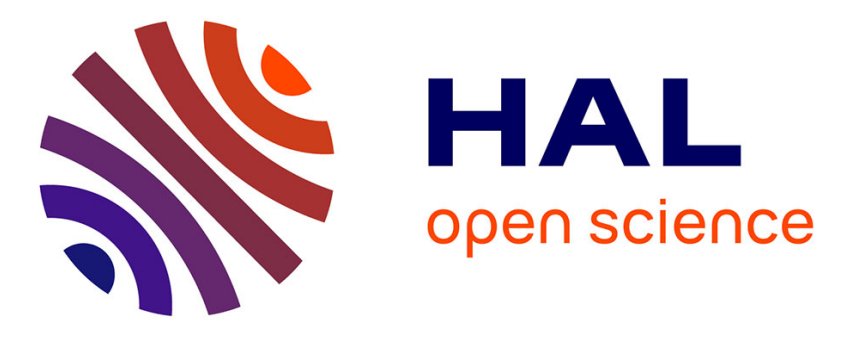

\title{
Chemically grafted of single-walled carbon nanotubes onto a functionalized silicon surface
}

Haythem Gammoudi, Fatma Belkhiria, Saloua Helali, Ben Assaker, Ibtissem Gammoudi, Fabien Morote, Ahmed Souissi, Mokhtar Karyaoui, Mosbah Amlouk, Touria Cohen-Bouhacina, et al.

\section{To cite this version:}

Haythem Gammoudi, Fatma Belkhiria, Saloua Helali, Ben Assaker, Ibtissem Gammoudi, et al.. Chemically grafted of single-walled carbon nanotubes onto a functionalized silicon surface. Journal of Alloys and Compounds, 2017, 694, pp.1036 - 1044. 10.1016/j.jallcom.2016.10.123 . hal-01397395

\section{HAL Id: hal-01397395 \\ https://hal.science/hal-01397395}

Submitted on 15 Nov 2016

HAL is a multi-disciplinary open access archive for the deposit and dissemination of scientific research documents, whether they are published or not. The documents may come from teaching and research institutions in France or abroad, or from public or private research centers.
L'archive ouverte pluridisciplinaire HAL, est destinée au dépôt et à la diffusion de documents scientifiques de niveau recherche, publiés ou non, émanant des établissements d'enseignement et de recherche français ou étrangers, des laboratoires publics ou privés.

\section{()(1)(2)}

Distributed under a Creative Commons Attribution - ShareAlikel 4.0 International 


\title{
Chemically grafted of single-walled carbon nanotubes onto a functionalized silicon surface
}

\author{
Haythem Gammoudi a, *, Fatma Belkhiria a, Saloua Helali a , Ibtissem Ben Assaker ${ }^{\text {a }}$, \\ Ibtissem Gammoudi ${ }^{\text {b }}$, Fabien Morote ${ }^{\mathrm{b}}$, Ahmed Souissi ${ }^{\mathrm{a}}$, Mokhtar Karyaoui ${ }^{\mathrm{a}}$, \\ Mosbah Amlouk ${ }^{c}$, Touria Cohen-Bouhacina ${ }^{\mathrm{b}}$, Radhouane Chtourou ${ }^{\mathrm{a}}$ \\ ${ }^{a}$ Université Tunis el Manar, Laboratoire de nanomatériaux et des systèmes pour les énergies renouvelables, Centre de Recherches et des Technologies de \\ l'Energie, BP. 95, Hammam Lif 2050, Tunisia \\ ' LOMA, Laboratoire Ondes et Matiere d'Aquitaine Université de Bordeaux, 351, cours de la libération, 33405 Talence Cedex, France \\ ${ }^{c}$ Unité de physique des dispositifs à semi-conducteurs, Faculté des sciences de Tunis, Université Tunis El Manar, 2092 Tunis, Tunisia
}

Keywords:

Single-walled carbon nanotubes

3-Aminopropyltriethoxysilane

Porous pyramidal silicon

\begin{abstract}
A B S T R A C T
This paper highlights some interesting results regarding the Single walled carbon nanotubes (SWCNTs) functionalized by carboxylic acid, which were dispersed with ultrasonic vibration and implanted using 3 aminopropyltriethoxysilane (APTES) solution on a porous pyramidal silicon (PPS) surface. The physical characterizations of the samples were studied by both Scanning Electron Microscopy (SEM) and Atomic Force Microscopy (AFM). Also, Fourier transform infrared spectroscopy (FTIR) has been carried out. First, SEM morphological observations reveals that nano pores dig into the sides of the micro pyramids and SWCNTs located throughout the area Si pyramidal structure. Second, AFM confirm the information ob tained by various other techniques such those of electron microscopy. Third, Fourier transform infrared spectroscopy (FTIR) analysis confirms the chemical bonding between the silicon and SWCNTs. Finally, a graphical abstract is proposed to describe the implantation steps of SWCNTs on the porous silicon (pSi) having pyramidal structure.
\end{abstract}

\section{Introduction}

Durant the last decade, the carbon nanotubes (CNTs) have emerged as new materials with a variety of potential applications [1] [2], and [3]. This is due to their remarkable mechanical, thermal, optical and electrical properties.

However, many of these interesting and unique properties can only be realized once the CNTs are integrated into more complex assemblies: CNTs/Nano composites applications [4] [5], and [6], particularly in the field emission, solar cells, electronics domains and enhanced functional materials [7] and [8]. The use of SWCNTs in the majority of these fields requires control over their placement and coverage on a surface, because such surfaces on CNTs play an important role to enhance their performance [9]. Also, it is reported that pSi seems an ideal biomaterial due to its biodegradability, large internal surface area and biocompatibility, which leads to many

\footnotetext{
* Corresponding author. Tel.: +216 50884993.

E-mail address: haythemgammoudi@hotmail.fr (H. Gammoudi).
}

applications such as: in biosensor design, tissue engineering and drug delivery [10].

One particular approach for implementing these properties is the formation of two scale structured surfaces, micron scale structured surfaces that also possesses nanometer scale structure and the attachment of SWCNTs in this specific surface.

The first tries to attach SWCNTs in specific surface was in 2000 with the dicyclohexylcarbodiimide (DCC), mediated assembly of CNTs onto a surface when Liu et al. [11] attached shortened SWCNTs to gold. In such work, the amine group forms a cysteamine mole cule which is used to create an amide linkage to the carboxylic acid on SWCNTs. The remaining thiol group on cysteamine was then used to attach to the gold substrate. The same authors used the AFM to visualize the SWCNTs Au surfaces where they observed needle like protrusions which were described as SWCNTs perpen dicularly to the substrate [11]. This initial report was followed up in 2001 by another study in which they described the alignment of carboxylic acid functionalized SWCNTs on silver. Also, they re ported that the negatively charged carboxylic acid groups were electrostatically attracted to the positively charged silver surface 
[12]. Since that time, many attempts have been published, with CNTs assembled on gold using various amine terminated thiol monolayers with the predominant application of electrochemical sensing [13], [14].

Nevertheless, carbon nanotubes were not chemically attached to silicon until 2006 when Yu et al. [15] attached shortened SWCNTs to silicon by first hydrogenating a silicon (100) surface and then reacting with ethyl undecylenate to reach an ethyl undeca noate self assembled monolayer. The ester terminal of the mono layer was reduced to an alcohol whereupon the shortened carboxylated SWCNTs were covalently attached using DCC coupling. It was found from AFM observations that vertically aligned SWCNTs were attached to the surface. Cyclic voltammetry of the surfaces revealed that the SWCNTs to silicon electronic connection was not strong, indicating that the self assembled Monolayer (SAM) hindered electron transduction.

In 2007, Yu et al. [16] refined chiefly the approach by hydrox ylating silicon (100) surfaces and then chemically attaching short ened SWCNTs using DCC coupling. The SWCNTs arrays formed were shown to be oriented vertically and cyclic voltammetry of the surfaces showed that the surface was suitable for electronic ap plications. In the same period, Flavel et al. [17] attached SWCNTs to an amine terminated silane layer (APTES) on silicon. This method produced well aligned SWCNT arrays using appropriate reaction between an amine and carboxylic acid to produce a stable amide linkage. Vertically aligned CNTs arrays produced by wet chemical assembly have been tested as chemical sensors [16], biological sensors [14], and photovoltaic devices [18] and [19] where they have shown promise as a simple method of producing functional surface bound CNTs arrays.

In 2010, Cameron et al. [7] reported both the chemical attach ment of functionalized SWCNTs to amino silane modified pSi and the formation of silane patterns on $\mathrm{pSi}$. In this context, their results show that pSi pore sizes and porosity increase across the substrate, SWCNTs coverage decreases concurrently, and their observations confirm that with decreasing APTES density on the surface, the coverage of the attached SWCNTs also decreases. In 2012, the same authors used pSi functionalized with APTES to immobilized carboxylated SWCNTs [8]. They found that the CNTs surface coverage noticeably altered the field emission properties of the surface. Also, their work covered the fact that there was an optimal coverage at which point there is enough CNTs to produce a large amount of current proving that CNTs surface coverage has also been found to have a profound effect on solar cell performance, cell adhesion and thin film transistors. By 2013, the same researchers found that both the chemical attachment and patterning of SWCNTs on pSi exhibit the ability of SWCNTs to capture human neuroblastoma cells [20].

The novelty in this work is indeed to modify the silicon topog raphy by a PPS structure [21] and [22] to increase the surface contacted with the carbon nanotube; and then attached chemically SWCNTs, on PPS surface and into the nano pores of PPS structure.

Indeed, the problem that persists is the implantation of carbon nanotubes on PPS surface and into the nano pores of PPS structure, which imposes to investigate the pore and pyramid size adapted to the size of SWCNTs.

Micron scale structured surfaces can being done by using anisotropic etching generally undertaken by using sodium hy droxide $(\mathrm{NaOH})$, a pyramidal form of silicon was obtained during this chemical attack. Furthermore, a chemical etching time opti mization is necessary to obtain a good distribution of all pyramids along the surface of Si [23] [24], and [25].

Nanometer scale structure surfaces can being done by using electrochemical etching in HF solutions diluted with ethanol [26] [27], and [28] to forming the nano porous coating on wafers of $\mathrm{Si}$ pyramids pre textured.

In the present work, an efficient method is provided to control the pore size using a circular electrode that covers the entire sample surface to ensure the homogeneity of these pores and their sizes.

For the implantation of carbon nanotubes on the PPS, we chose the silanization of SWCNTs functionalized by carboxylic acid $-\mathrm{COOH}$ with a coupling agent APTES to enhance the interfacial adhesion between nanotubes and the matrix of the PPS surface and to help to the dispersion and homogeneous implanting of the SWCNTs on the same surface [29] and [30]. This silanization method of SWCNTs allows us to control both the time and the suitable quantities used as well as concentration [7].

\section{Experimental details}

\subsection{Materials}

Single wall carbon nanotubes (SWCNTs) (Fig. 1), containing carboxylic acid functionality were purchased from Sigma Aldrich. The diameter range of the SWCNTs was $4-5 \mathrm{~nm}$, the length was $0.5-1.5 \mu \mathrm{m}$ and the purity is above $90 \%$.

APTES (Fig. 1): with purity of 99\% (Aldrich) was used for attached functionalized SWCNTs with a carboxylic acid on a PPS structure (Fig. 1). The following reagents were used without further purification.

\subsection{Substrates preparation}

$P$ type $\left(\begin{array}{lll}1 & 0 & 0\end{array}\right)$ oriented silicon wafers were employed for the experiment. The resistivity of the samples was between 0.01 and $0.02 \Omega \mathrm{cm}$. The size of samples was $20 \times 20 \pm 1 \mathrm{~mm}$. First, the wafers were firstly washed by acetone, ethanol, deionized water and iso propanol. After that, these wafers were immersed in $\mathrm{NaOH}(1 \mathrm{M})$ solution at $85{ }^{\circ} \mathrm{C}$ during 6 min to remove Si damage [31] and [32].

Pyramidal silicon structures were formed after chemical etching; the silicon wafers were rinsed with plentiful deionized water and dried by blowing Nitrogen gas. Second, porous pyramids structured silicon layers were prepared by electrochemical anod ization of heavily electrolyte solution of $\mathrm{HF}(25 \%)$ and $\mathrm{EtOH}$, respectively. The anodization cell is a Teflon beaker where the c $\mathrm{Si}$ wafer acts as the anode and the cathode is formed by a platinum wire [3] [26], and [33]. Finally, a computer controlled current source was used to fabricate porous pyramids structured silicon from selected current density and time data, as shown in Table 1.

After the electrochemical attacks, all the samples were rinsed with ethanol and dried in the ambient air for the formation of an oxide layer $\mathrm{SiO}_{\mathrm{x}}$ [34] [35] [36], and [37].

Dispersion and implantation steps of SWCNTs were done on three steps. First step consists on the dispersion of SWCNTs in a $25 \mathrm{ml}$ volumetric flask, carbon nanotubes $(1 \mathrm{mg})$ were mixed with Diméthylformamide (DMF, $10 \mathrm{ml}$ ). It was then sealed with a Teflon cap and dispersed via ultra sonication for 30 min until the mixture was visually homogeneous (Fig. 2).

Second step concerns the use of the coupling agent APTES which was prepared with different concentrations of $1 \%$ and $5 \%$ in the mixtures with ethanol [38]; this solution was made using a $100 \mathrm{ml}$ Teflon beaker. Each sample of PPS structure emerged from $8 \mathrm{~h}$ in the various concentrations of APTES as shown in Table 1, then rinsing with ethanol to remove the APTES that has not been attached to the surface of PPS structure. This treatment leads to the formation of APTES network, which progressively cover the inside of the pores and the entire porous surface.

In third step, the solution of dispersed SWCNTs was deposited by spray method on different PPS structure substrates with 


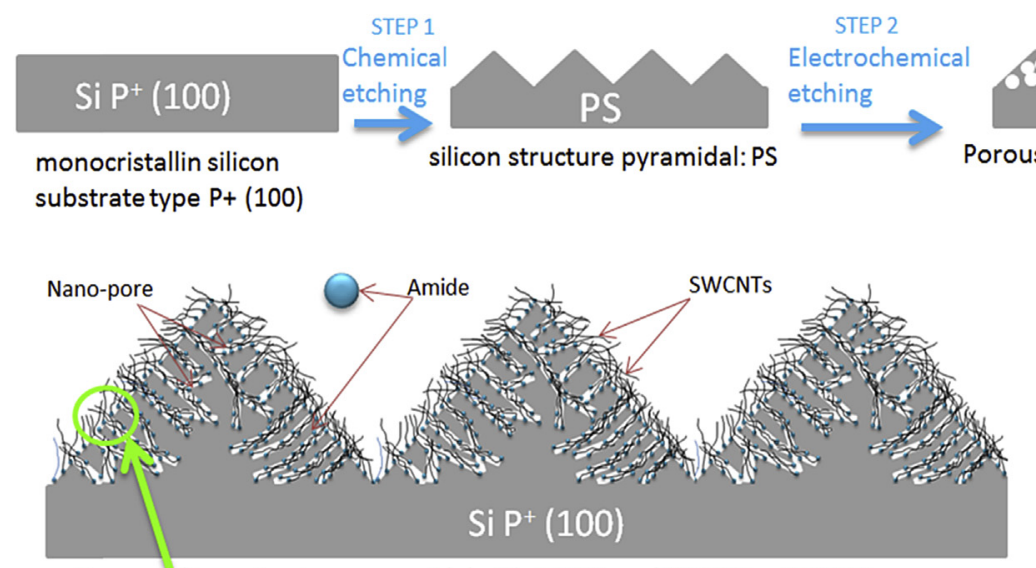

Porous silicon structure pyramidal with APTES and SWCNTs : CNPPAS
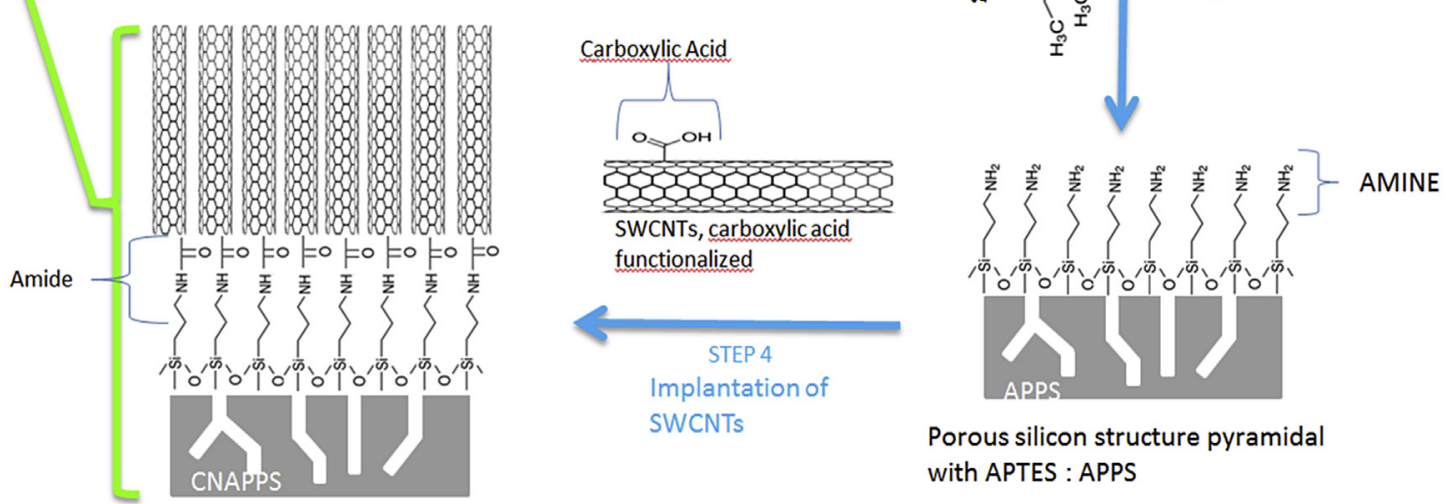

Implantation of

SWCNTS

Porous silicon structure pyramidal with APTES : APPS

Porous silicon structure pyramidal with APTES and SWCNTs : CNPPAS

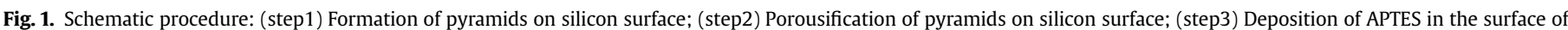
silicon porous structured pyramidal; (step4) Deposition of carbon nanotubes on silicon porous structured pyramidal surface with APTES. Substrates preparation.

Table 1

Preparation parameters of the porous pyramidal silicon structures and concentration of APTES and SWCNTs.

\begin{tabular}{|c|c|c|c|c|c|}
\hline \multirow[t]{2}{*}{ Samples } & \multirow[t]{2}{*}{ Chemical etching (min) } & \multicolumn{2}{|c|}{ Electrochemical etching } & \multirow[t]{2}{*}{ Concentration of APTES (\%) } & \multirow[t]{2}{*}{ Concentration of SWCNTs (M) } \\
\hline & & Current (mA) & Time (S) & & \\
\hline PS & 6 & & & & \\
\hline PPS & 6 & 60 & 35 & & \\
\hline APPS1 & 6 & 60 & 35 & 1 & \\
\hline APPS5 & 6 & 60 & 35 & 5 & \\
\hline CNAPPS1 & 6 & 60 & 35 & 1 & 1 \\
\hline CNAPPS5 & 6 & 60 & 35 & 5 & 1 \\
\hline
\end{tabular}

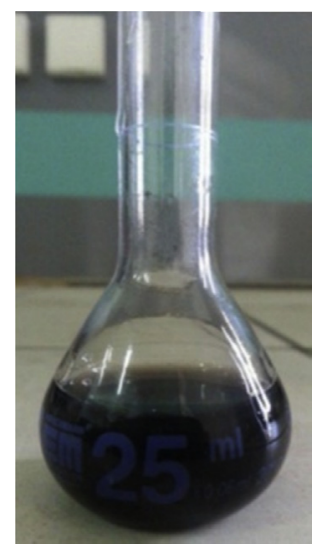

Fig. 2. Dispersion of SWCNTs in a volumetric flask. different concentrations of APTES. The obtained samples were heated for $1 \mathrm{~h}$ at $120{ }^{\circ} \mathrm{C}$ for drying and tailings disposal. The fabrication process of all the samples step is schematically shown in Graphical abstract (Fig. 3).

\subsection{Characterization techniques}

Fourier Transform infrared spectra were recorded in trans mittance mode at room temperature from 350 to $4000 \mathrm{~cm}^{-1}$ with a Fourier Transform infrared spectrometer (Perkin Elmer Spectrum 1000). The morphology of the samples was characterized with HITACHI S 4800 scanning electron microscopy (SEM) and Bioscope II, Bruker, Atomic Force Microscope (AFM). AFM is a high resolution scanning probe surface analysis technique, based on the measure ment of interaction forces between a fine tip and the sample sur face, these forces depending on the tip sample distance [39] and [40]. AFM images were recorded with a BioscopeII AFM setup 

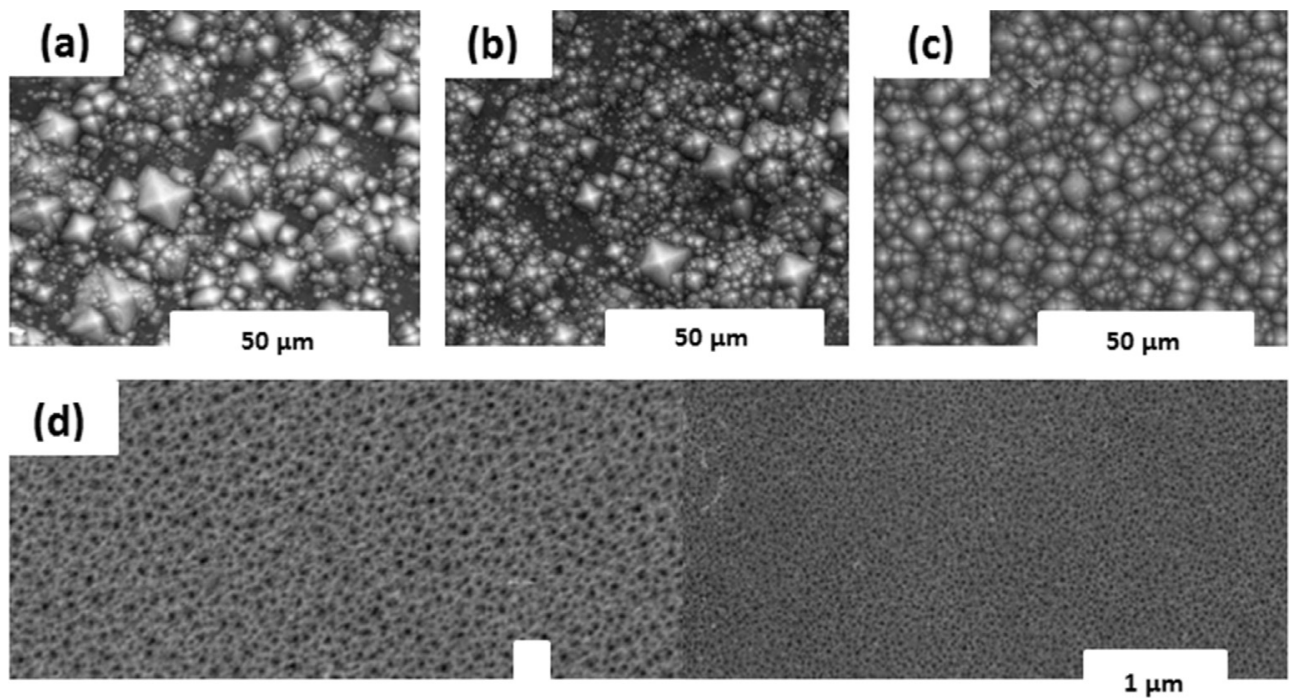

$100 \mathrm{~nm}$

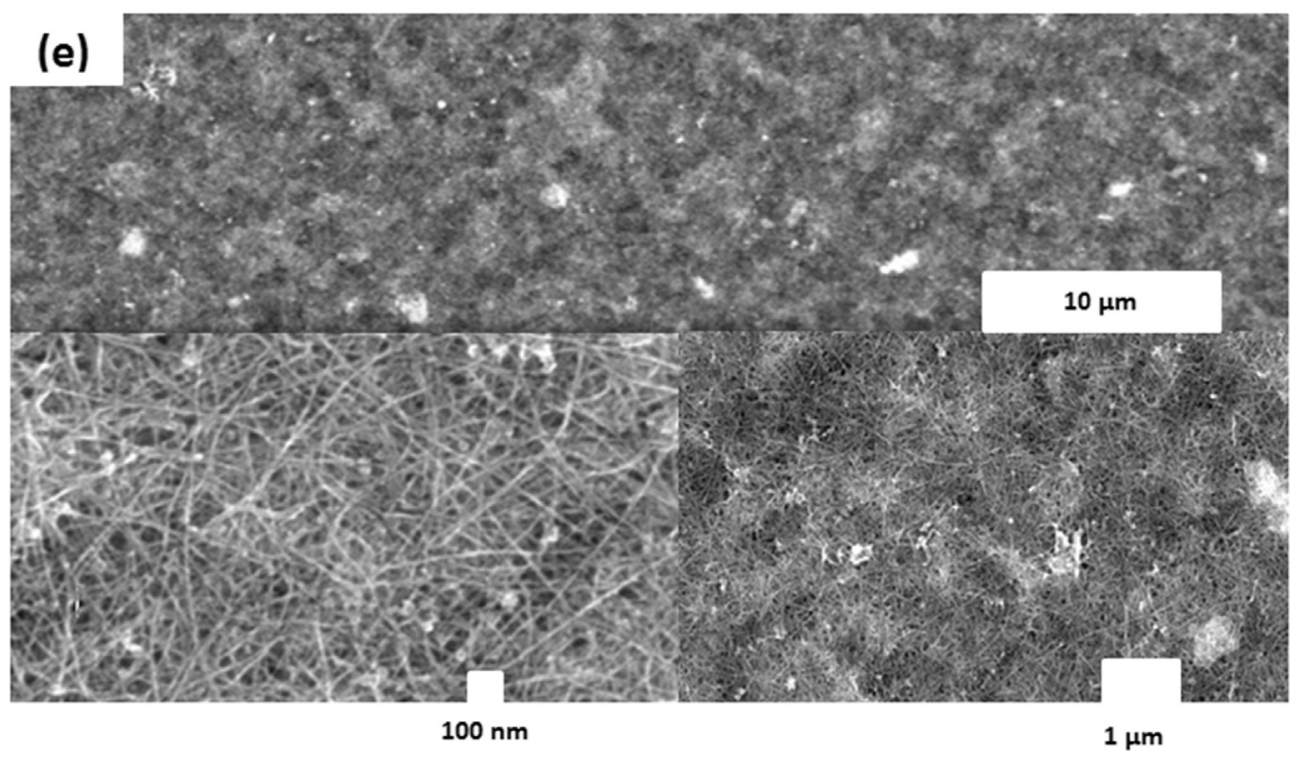

Fig. 3. SEM Images of: (a) Pyramidal silicon structure (PS), (b) Porous pyramidal silicon structure (PPS), (c) Porous pyramidal silicon structure with APTES (APPS), (d) Porous pyramidal silicon structure with a low-scale view and a large-scale view (PPS), (e) Porous pyramidal silicon structure with APTES and SWCNTs with a low-scale view and a largescale view (CNAPPS).

(Veeco Brucker, Santa CA) equipped with a G scanner (maximum $\mathrm{XYZ}$ scan range of $150 \mu \mathrm{m} \times 150 \mu \mathrm{m} \times 12 \mu \mathrm{m})$. Samples were scanned in tapping mode using PPP NCL silicon probes (NANO SENSORSTM) with a spring constant of about $35 \mathrm{~N} \mathrm{~m}^{-1}$ and a cor responding measured resonance frequency of about $165 \mathrm{kHz}$. All scans were done with scan rates between $0.3 \mathrm{~Hz}$ and $1 \mathrm{~Hz}$ (ac cording to the scan size and the scanning mode). AFM data were processed using the Nanoscope (version 7.30, Veeco).

\section{Results and discussion}

\subsection{SEM morphological study}

Fig. 3 shows the SEM results obtained on realized structure. Due to anisotropic etching of mono crystalline silicon with $\mathrm{NaOH}$ so lution, the whole wafer surface was homogeneously covered (85\% coverage) by pyramidal structure of silicon (PS) (Fig. 3a).

Furthermore, the sizes of the pyramids are not uniform
( $2 \mu \mathrm{m}-7 \mu \mathrm{m})$, the silicon surface with an average pyramid size of $4.5 \mu \mathrm{m}$ and the facets of the pyramids are all smooth.

This microstructuring of silicon provides an increase in the surface area with keeping the same size of samples, which make it an ideal biomaterial in terms of acceleration of their biodegrad ability [20].

Then the silicon wafers where electrochemically etched to form multilayered PPS on the pyramids. It is generally assumed that pore initiation occurs at surface active sites defects or irregularities. The properties of PPS layer (porosity, thickness, pore diameter and microstructure) mainly depend on the operating parameters of layer formation, including the HF concentration ratio, the presence of surface active compound additives, the duration and the tem perature as well as the wafer type and its resistivity [7]. The in vestigations have been focused on visualization techniques, because the optical, electrical and physical properties of PPS layer depend strongly on its microstructure.

Fig. 3b, d displays the optical micro and nano imagery of PPS 
samples with low and high magnification. It can be seen that the surface of Si wafer with PPS shows black color (Fig. 3b) comparing to Fig. 3a, which indicates much lower reflectance of the silicon surface. When the magnifications are low, only pyramids could be observed (Fig. 3b). As the magnification increases, one can see that the porosification was already fabricated on every facet of pyra mids, which are distributed uniformly around the whole substrate, (Fig. 3d). The diameters of the PPS pores are identical and their sizes are around $20 \mathrm{~mm}$ as shown in the zoom of Fig. 3d (for the sake of clarity). So, it can be noted that the PPS multilayer is nano structured, and the pyramids remain unchanged after the electro chemical etching. Thus, they are clearly observed in the plan view (Fig. 3b). The obtained porosity was about $80 \%$ which is in good agreement with the result found elsewhere [20].

\subsection{AFM morphological study}

We used AFM to confirm and also to supplement the informa tion obtained by various other techniques such those of electron microscopy.

As shown in Fig. 4, the physico chemical treatment of silicon with APTES has been effective. Indeed, the silanized sample has a relatively rougher surface than that of the untreated substrate [7] and [41], the grain size is slightly larger and also note the pres ence of many aggregates. The average roughness is about $14 \mathrm{~nm}$ for the PPS structure (Fig. 4b) and $3 \mathrm{~nm}$ for the silanized surface (Fig. 4d).

Similarly, in the case of the study of the Chemical fixation of SWCNTs to an amine terminated silane (APTES), AFM results confirm those obtained by SEM. So, AFM imaging was completed for three samples at different concentrations of APTES, $0 \%, 1 \%$ and
$5 \%$ to determine their influence on the bonding strength and the surface coverage. As shown in Fig. 5, the deposition of nanotubes for untreated surface is not very dense (Fig. 5 a, b, c). The contrast of the corresponding height image (Fig. $5 \mathrm{a}, \mathrm{c}$ ) is not well resolved because the deposit nanotubes are not very dense [7] and [41], and more, the grain size of the silicon surface are of the same order of magnitude as those of the nanotubes. The "derived signal image" (Fig. 5b) highlights the sub structures such as nanotubes or grains of the reference substrate and gives also a "3D view" of the topography.

As shown in Fig. 5 e, f, g one can make the same observation for the surface silanized with a low APTES concentration (1\%). In this case, the nanotubes are well deposited but with no preferred orientation.

However, for intermediate concentration of APTES (between 1 and $5 \%$ ), the surface can be completely covered can be well covered but the nanotubes are not well attached. But, once we begin to scan the surface, the tip gets polluted, possibly by clinging to the tip SWCNTs which is causing duplication of nanotubes observed in the corresponding AFM images and that complicates the character ization (results not shown here). On the other hand, the contrast is striking for the concentration of APTES of 5\%, the SWCNTs density is high and the surface is completely covered by well deposited entangled nanotubes as observed in Fig. $5 \mathrm{~g}$, h, i So, it is obvious that treatment with silane optimizes the recovery by the nanotubes and ensures their good adhesion. This finding regarding the implanta tion of CNTs is consistent with the one reported by Cameron et al. [7].

We conclude that the increase in surface coverage is tributary to an increase in reactive surface sites and the formation of the more favorable amide bond, while the entangled is most likely due to the
PPS
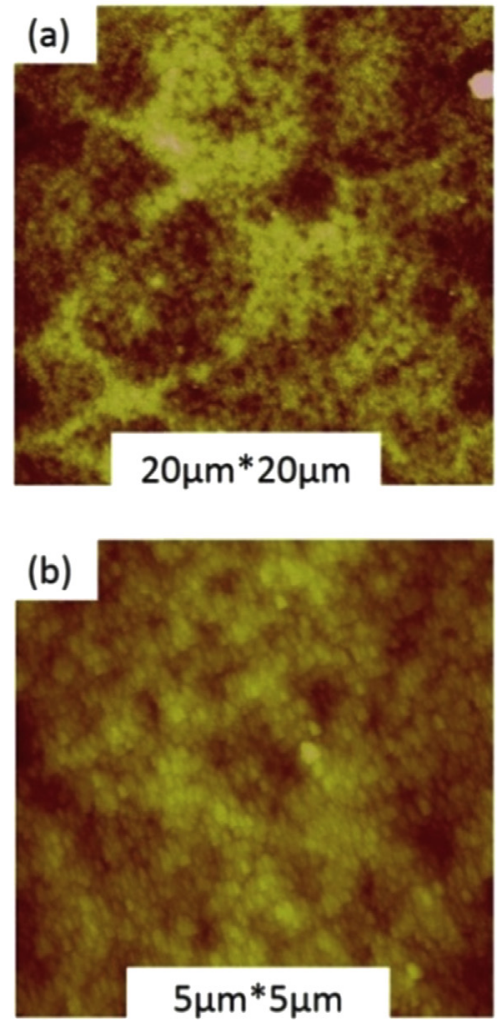

$200 \mathrm{~nm}$
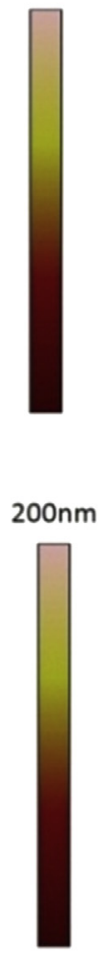

APPS
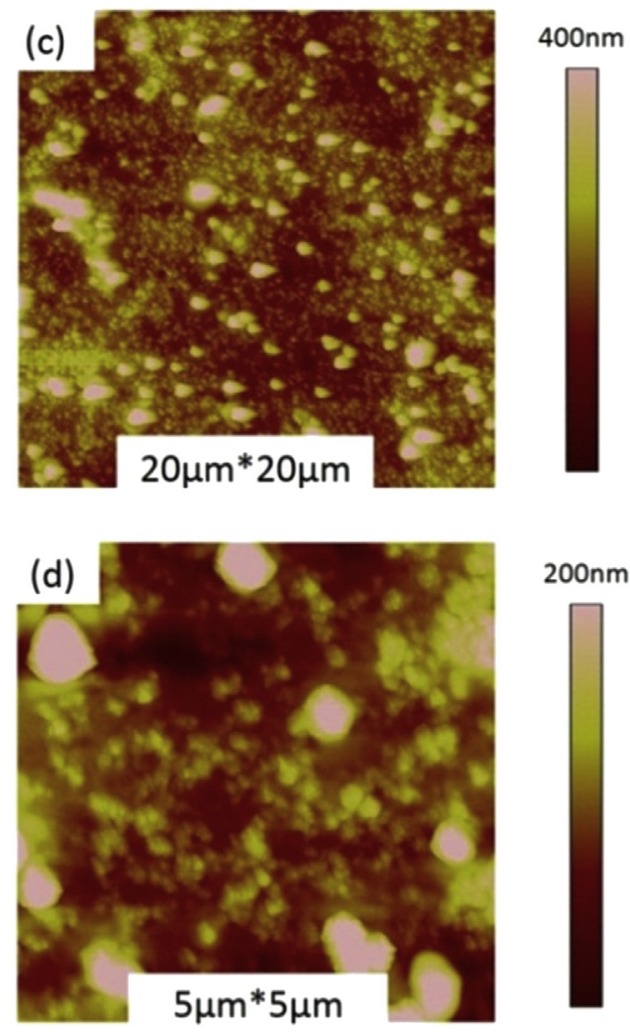

$200 \mathrm{~nm}$

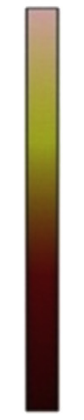

Fig. 4. AFM Height image of: (a,b) Porous silicon structure (PPS); (c,d) Porous silicon structure with APTES (APPS). 
Structured porous silicon non functionalized with APTES
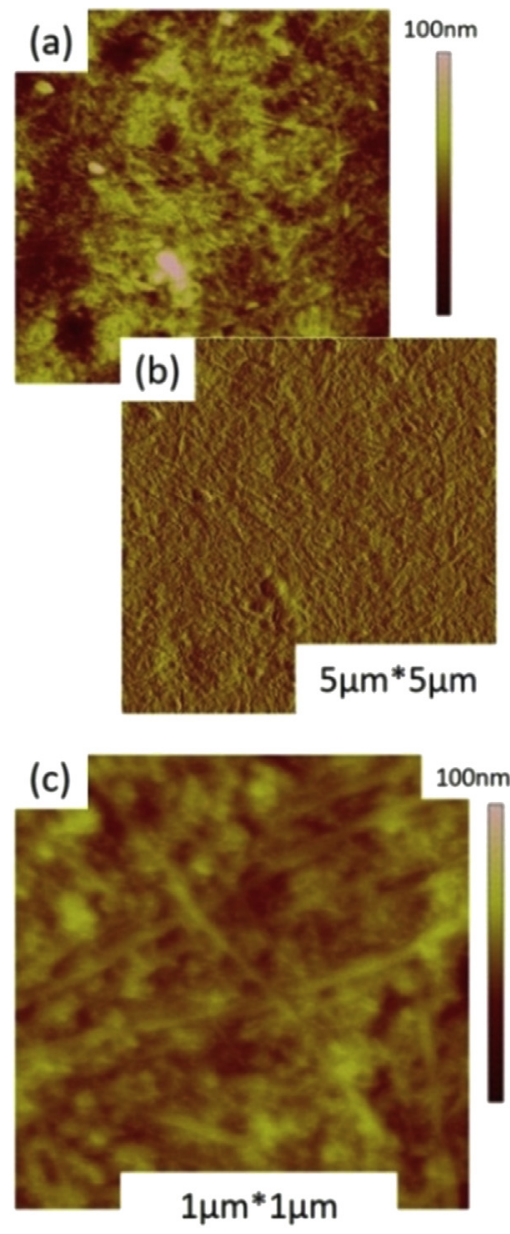

Structured porous silicon functionalized with APTES - Low concentration (1\%)
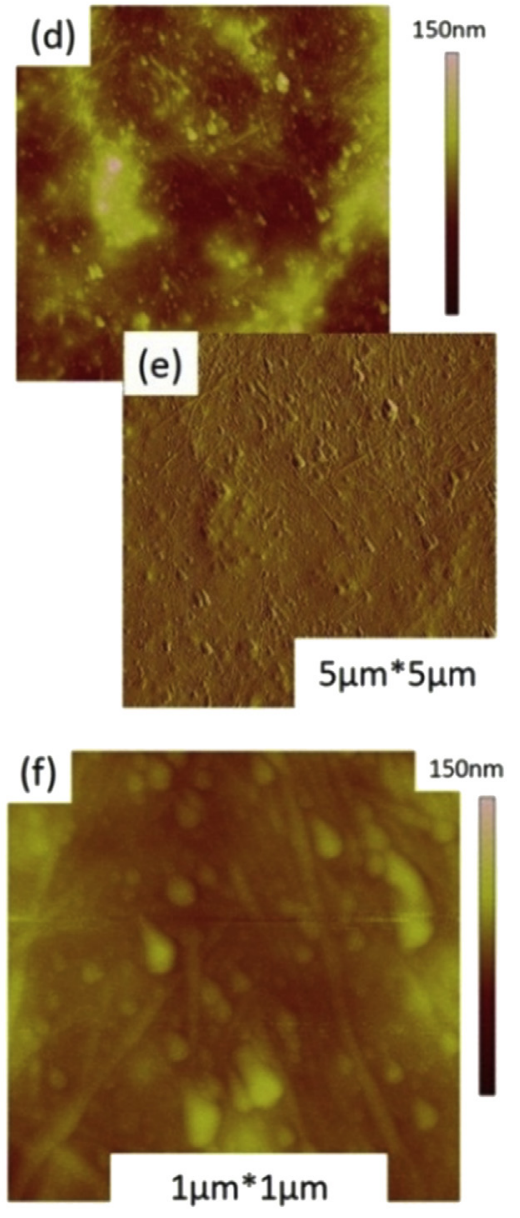

Structured porous silicon functionalized with APTES - High concentration (5\%)
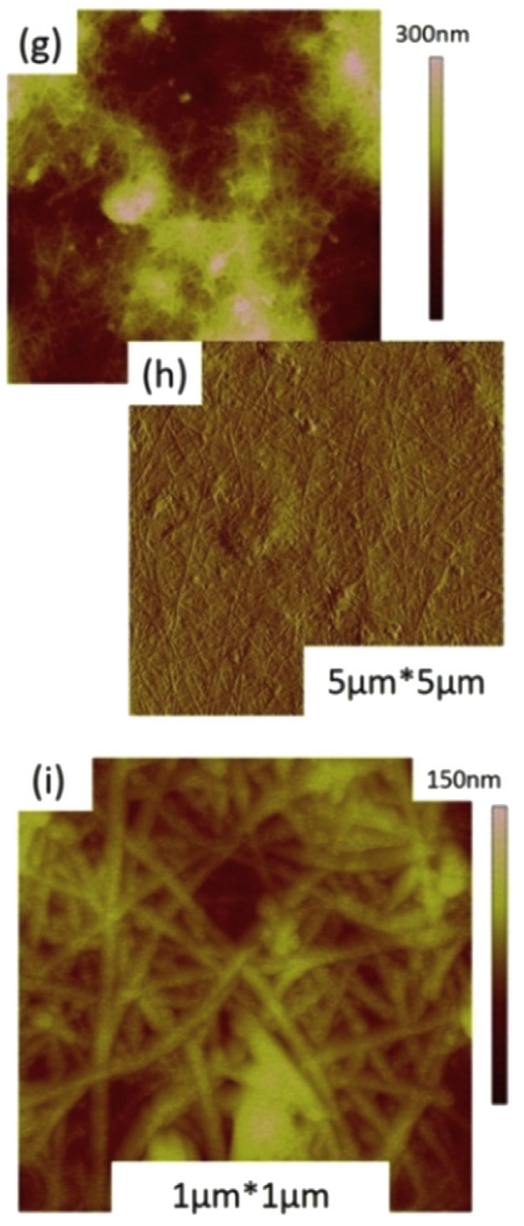

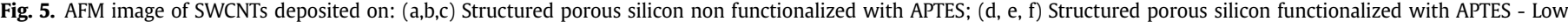
concentration (1\%); (g, h, i) Structured porous silicon functionalized with APTES - High concentration (5\%); (a, c, d, f, g, i) Height images; (b, e, h) derived signal.

less hydrophilic APTES functionalized surface [7] [10], [20] [41] [42], and [43].

\subsection{FTIR structural study}

Fig. 7 shows FTIR spectra of the realized structures. To increase the coverage of SWCNTs on the silicon surface, a PPS surface is synthesized in which an oxide layer $\mathrm{SiO}_{2}$ was formed during the drying time in ambient air (Fig. 6a), because the instability of $\mathrm{Si}-\mathrm{H}$ bonds, a layer of APTES was deposited to produce an amine terminated monolayer (Figs. 3, 4b and 6) that reacts with the car boxylic acid attached at SWCNTs (Figs. 3 and 6) that forms (or leading to) an amide layer (Figs. 3, 4c and 6). In the case of Fig. 7a, the PPS surface displays characteristic $\mathrm{Si}-\mathrm{H}$ vibrational modes at

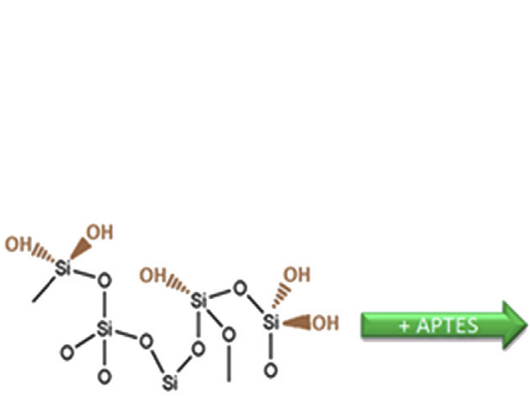

(a)

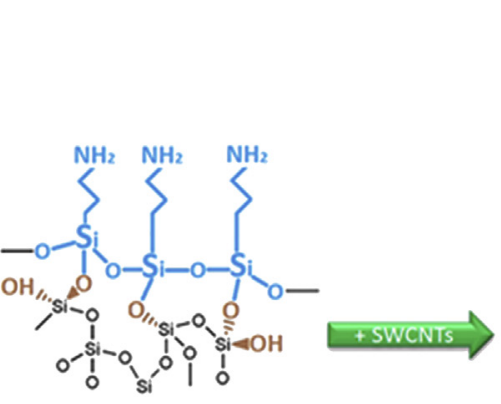

(b)

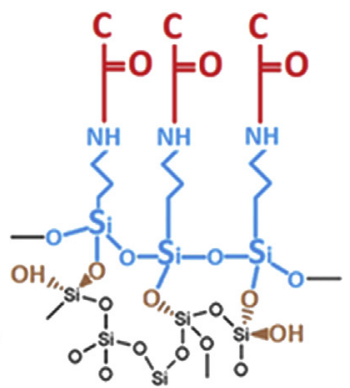

(c)

Fig. 6. Schematic representation of (a) oxidized Si (b) oxidized Si after amine immobilization and (c) oxidized Si after amine immobilization and carboxylic acid attachment. 


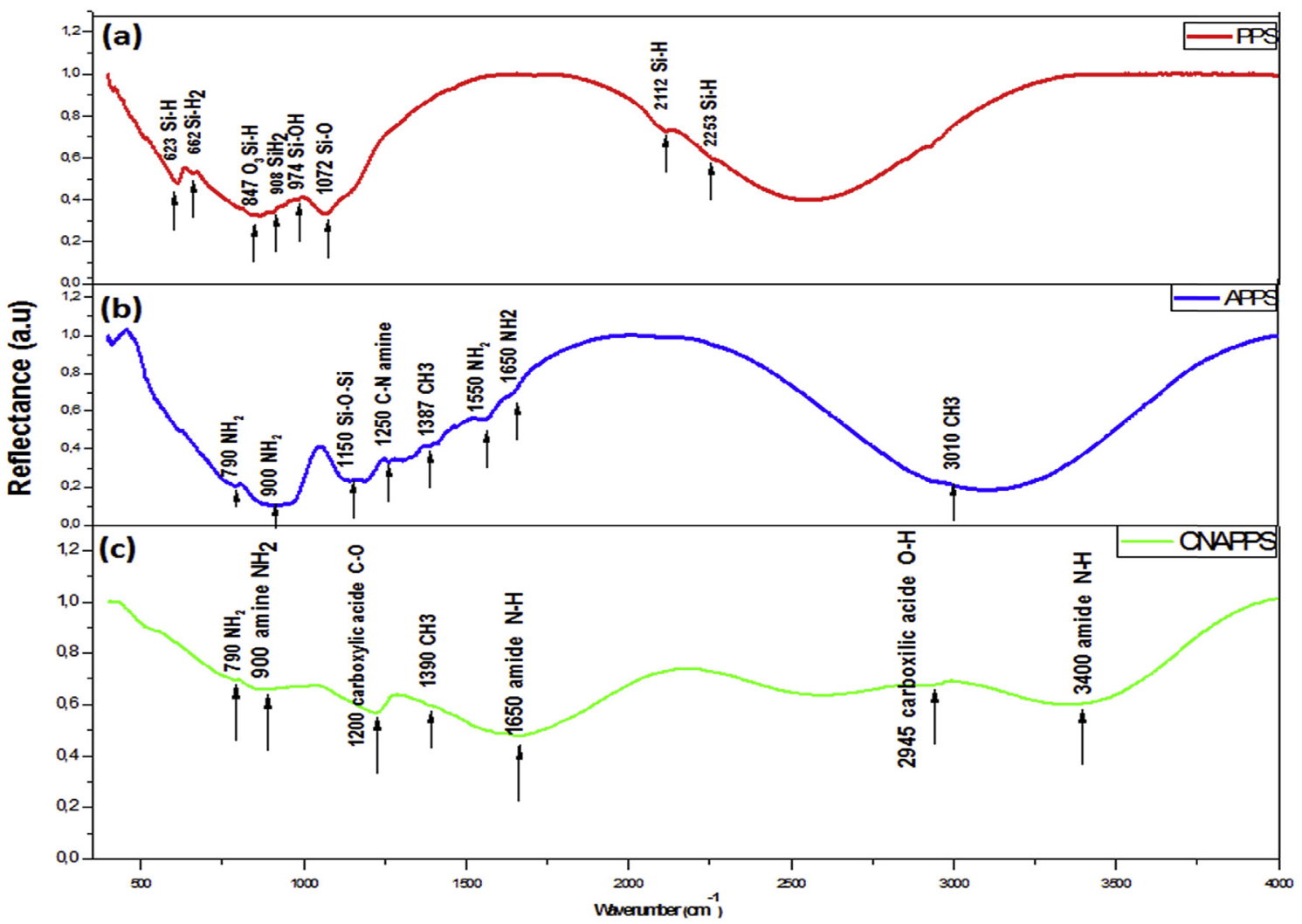

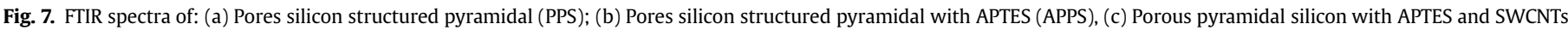
structure (CNAPPS).

$623,662,908,2112$ and $2253 \mathrm{~cm}^{-1}$ corresponding to stretching and binding vibration modes, and shows the formation of the peaks corresponding to vibrations of the silicon oxide ( $\mathrm{Si}-\mathrm{O}-\mathrm{Si}$ ) located at $1072 \mathrm{~cm}^{-1}$ [44] [45], and [46], A weak oxidation peak (silanol) assigned to $974 \mathrm{~cm}^{-1}$ is attributed to $\mathrm{Si}-\mathrm{OH}$ groups which facili tates the fixation of APTEs on the PPS surface [47] and a peak at $874 \mathrm{~cm}^{-1}$ is attributed to O3Si-H wagging mode.

Once the APTES surface has been filed (Fig. 7b), we observe the degradation of $\mathrm{Si}-\mathrm{H}_{\mathrm{x}}$ bonds due to their instability and a slight increase in the oxidation of $\mathrm{Si}-\mathrm{O}-\mathrm{Si}$ during the time. Moreover, a broad peak appearing at $900 \mathrm{~cm}^{-1}$ is attributed to $\mathrm{N}-\mathrm{H}_{2}$ scissoring instead of the low oxide peak $\mathrm{SiO}-\mathrm{H}$ at $974 \mathrm{~cm}^{-1}$, and the one located at $1550 \mathrm{~cm}^{-1}$ corresponds to $\mathrm{N}-\mathrm{H}_{2}$ bending [48]. In the same line, three small primary amine peaks assigned at 790, 1250 and $1650 \mathrm{~cm}^{-1}$ are related to the urethane bond within the APTES [49] [50], and [51] On the other hand, there is also a broad vibra tional peak around $3010 \mathrm{~cm}^{-1}$ attributed to the terminal $\mathrm{CH}_{3}$ group of the APTES. Also, it is found that the silicon oxide ( $\mathrm{Si}-\mathrm{O}-\mathrm{Si}$ ) stretching (bending) vibrational peak at $1150 \mathrm{~cm}^{-1}$ still exists but with low intensity.

We deduce then, that after the filing of APTES there is the appearance of the amine groups in the PPS surface that promotes binding of SWCNTs. The final FTIR spectrum displayed in Fig. 7c corresponds to SWCNTs deposited in PPS with APTES surface. Upon SWCNTs attachment (Fig. 7c), the amine peaks at around 1250 , 1550 and $1650 \mathrm{~cm}^{-1}$ disappear, $\mathrm{C}-\mathrm{H}$ stretching peaks remain and two new peaks appear at $1200 \mathrm{~cm}^{-1}$ and $2945 \mathrm{~cm}^{-1}$ which are attributed to the carboxylic acid groups $(\mathrm{O}=\mathrm{OH})$ attached on the surface of SWCNTs (Fig. 1). The large bands at 790 and $850 \mathrm{~cm}^{-1}$ with low intensity is also indicates that the chemical functional amine $\left(\mathrm{NH}_{2}\right)$ groups associated with each APTES are reserved after deposition of SWCNTs which returns that the amount of amine does not react with all the acid carboxylic. Two new bands appeared at $1200 \mathrm{~cm}^{-1}$ and $3400 \mathrm{~cm}^{-1}$ corresponding to the amide bond thus confirms the reaction of amine with carboxylic acid group.

The FTIR spectra support the formation of an amine terminated monolayer and the creation of an amide linkage between the APTES and functionalized SWCNTs (Fig. 4), that confirms the successful implantation and dispersion of each SWCNTs into the PPS APTES surface. The higher coverage of SWCNTs observed, (Fig. 3e), is due to the favorable and stable amide linkage as well as the fact that the APTES layer does not include any germinal groups further increasing the stability of the attachment, and the APTES mediated attachment was considered the best method to produce SWCNTs PPS substrate.

\section{Conclusions}

In this work, we successfully constructed a composite structure combining pyramids, nano porosification, APTES and carbon nanotubes has been manufactured by both chemical and electro chemical etchings. Nano pores are formed by changing the time and the current of the electrochemical etching of a Si substrate structured pyramids. The optimal condition for the manufacture of this structure is obtained with a current of $60 \mathrm{~mA}$ and $35 \mathrm{~s}$ etching time. Single walled carbon nanotubes functionalized by carboxylic acid was silanized and implanted in two stages: First step consists of the surface preparation by direct immersion of PPS structure in APTES solution at room temperature and ambient pressure. Second step leads to the implantation of SWCNTs by spray method on PPS structure. 
Validation of PPS structure created and the chemical attachment of SWCNTs into this structure by APTES molecules were confirmed by AFM, SEM and FTIR.

Further investigation on $\mathrm{i}(\mathrm{v})$ characteristics as well as the thermal properties of such structures is in progress. Finally, this new combined structure may be of interest for various sensitivity applications such as gas sensors, waste water, biosensors and so on also, this study paves the way for possible use of this structure as chemical sensor functionalized by biological entity.

\section{Acknowledgments}

The authors gratefully acknowledge Dr. Guy Garry for giving us access to the Transmission Electron Microscope at Thales Research and Technology France, Palaiseau, France.

\section{References}

[1] D.A. Walters, L.M. Ericsson, M.J. Casavant, J. Liu, D.T. Colbert, K.A. Smith, R.E. Smalley, Elastic strain of freely suspended single-wall carbon nanotube ropes, Appl. Phys. Lett. 74 (25) (1999) 38033805.

[2] Y. Kobayashi, T. Yamashita, Y. Ueno, O. Niwa, Y. Homma, T. Ogino, Extremely intense Raman signals from single-walled carbon nanotubes suspended between Si nanopillars, Chem. Phys. Lett. 386 (1 3) (2004) 153157.

[3] Tae Jae Lee, Jungeun Seo, Haiwon Lee, Jung Woo Lee, Whikun Yi, Fabrication of single walled carbon nanotube three-dimensional networks inside the pores of a porous silicon structure, Carbon 48 (5) (2010) 14731479.

[4] L. Valentinia, J. Macanb, I. Armentanoa, F. Mengonia, Josè M. Kennya, Modification of fluorinated single-walled carbon nanotubes with aminosilane molecules, Carbon 44 (11) (2006) 21962201.

[5] Sander J. Tans, Michel H. Devoret, Hongjie Dai, Andreas Thess, Richard E. Smalley, L.J. Geerligs, Cees Dekker, Individual single-wall carbon nanotubes as quantum wires, Nature 386 (6624) (1997) 474477.

[6] P.G. Collins, K. Bradley, M. Ishigami, A. Zettl, Extreme oxygen sensitivity of electronic properties of carbon nanotubes, Science 287 (2000) 18011804.

[7] Cameron J. Shearer, Amanda V. Ellis, Joseph G. Shapter, Nicolas H. Voelcker, Chemically grafted carbon nanotube surface coverage gradients, Langmuir 23 (26) (2010) 1846818475.

[8] Cameron J. Shearer, Adam Fahy, Matthew Barr, Paul C. Dastoor, Joseph G. Shapter, Nanoscale Res.Lett. (2012) 2012720432.

[9] Leping Yu, Daniel D. Tune, Cameron J. Shearer, Joseph G. Shapter, Application of polymer interlayers in silicon carbon nanotube hetero junction solar cells, ChemNanoMat 1 (2015) 115121.

[10] Martin J. Sweetman, Cameron J. Shearer, Joseph G. Shapter, Nicolas $\mathrm{H}$. Voelcker, Dual silane surface functionalization for the selective attachmen of human neuronal cells to porous silicon, Langmuir 27 (2011) 94979503.

[11] Zhongfan Liu, Ziyong Shen, Tao Zhu, Shifeng Hou, Lizhen Ying, Organizing single-walled carbon nanotubes on gold using a wet chemical self-assembling technique, Langmuir 16 (8) (2000) 35693573.

[12] Bin Wu, Jin Zhang, Zhong Wei, Shengmin Cai, Zhongfan Liu, Chemical alignment of oxidatively shortened single-walled carbon nanotubes on silve surface, Phys. Chem. B 105 (22) (2001) 50755078.

[13] P. Diao, Z. Liu, Vertically aligned single-walled carbon nanotubes by chemical assembly methodology properties and applications, Adv. Mater. 22 (13) (2010) 14301449.

[14] J.J. Gooding, R. Wibowo, J. Liu, W. Yang, D. Losic, S. Orbons, F.J. Mearns J.G. Shapter, D.B. Hibbert, Protein electrochemistry using aligned carbon nanotube arrays, Am. Chem. Soc. 125 (2003) 90069007.

[15] Jingxian Yu, Dusan Losic, Matthew Marshall, Till Bocking, John Justin Gooding Joseph George Shapter, Preparation and characterization of an aligned carbon nanotube array on the silicon (100) surface, Soft Matter 2 (2006) 10811088

[16] J. Yu, J.G. Shapter, J.S. Quinton, M.R. Johnston, D.A. Beattie, Direct attachment of well-aligned single-walled carbon nanotube architectures to silicon (100) surfaces: a simple approach for device assembly, Phys. Chem. Chem. Phys. 9 (2007) 510520 .

[17] B.S. Flavel, J. Yu, J.G. Shapter, J.S. Quinton, Patterned attachment of carbon nanotubes to silane modified silicon, Carbon 45 (2007) 25512558.

[18] M.A. Bissett, J.G. Shapter, Photocurrent response from vertically aligned single-walled carbon nanotube arrays, J. Phys. Chem. C 114 (14) (2010) 67786783.

[19] J. Yu, S. Mathew, B.S. Flavel, M.R. Johnston, et al., Ruthenium porphyrin functionalized single-walled carbon nanotube arrays-a step toward light harvesting antenna and multibit information storage, Am. Chem. Soc. 130 (27) (2008) 87888796.

[20] Cameron J. Shearer, Frances J. Harding, Martin J. Sweetman, Joseph G. Shapter, Nicolas H. Voelcker, Nanostructured biointerfaces created from carbon nanotube patterned porous silicon films, Surf. Coat. Technol. 224 (2013) 4956.

[21] Jinsu Yooa, Gwonjong Yua, Junsin Yi, Black surface structures for crystalline silicon solar cells, Mater. Sci. Eng. 159160 (2009) 333337.

[22] Jianwei Shia, Fei Xu, Pinghua Zhou, Jie Yang, Zuobao Yang Dongsheng Chen, Yefei Yin, Dandan Chen, Zhongquan Ma, Refined Nano-textured surface coupled with $\mathrm{SiN}_{\mathrm{X}}$ layer on the improved photovoltaic properties of multicrystalline silicon solar cells, Solid State Electron. 85 (2013) 2327.

[23] H. Seidel, L. Csepregi, A. Heuberger, H. Baumgartel, Anisotropic etching of crystalline silicon in alkaline solutions, J. Electrochem. Soc. 137 (1990) 36123626.

[24] T. Juvonen, J. Harkonen, P. Kuivalainen, High Efficiency single crystalline silicon solar cells, Phys. Scr. T 101 (2002) 9698.

[25] Yujie Fan, Peide Han, Peng Liang, Yupeng Xing, Zhou Ye, Shaoxu Hu, Differences in etching characteristics of TMAH and $\mathrm{KOH}$ on preparing inverted, Appl. Surf. Sci. 264 (2013) 761766.

[26] Junfeng Xiao, Lei Wang, Xiaoqiang Li, Xiaodong Pi, Deren Yang, Reflectivity of porous-pyramids structured silicon surface, Appl. Surf. Sci. 257 (2010) 472475.

[27] K. Peng, J. Zhu, Morphological selection of electroless metal deposits on silicon in aqueous fluoride Solution, Electrochim. Acta 49 (2004) 25632568.

[28] N. Marrero, R. Guerrero-Lemus, B. González-Díaz, D. Borchert, Effect of porous silicon stain etched on large area alkaline textured crystalline silicon solar cells, Thin Solid Films 517 (2009) 26482650.

[29] D. Bethune, C. Kiang, M. De Vries, G. Gorman, R. Savoy, J. Vazquez, R. Beyers, Cobalt catalysed growth of carbon nanotubes with single-atomic-layer walls, Nature 363 (1993) 605607.

[30] V.N. Popov, Carbon Nanotubes properties and application, Mater. Sci. Eng. Rep. 43 (2004) 61102.

[31] Bohr-Ran Huang, Ying-Kan Yang, Wen-Luh Yang, Key technique for texturing a uniform pyramid structure with a layer of silicon nitride on monocrystalline silicon wafer, Appl. Surf. Sci. 266 (2013) 245249.

[32] Z. Xi, D. Yang, W. Dan, C. Jun, X. Li, D. Que, Investigation of texturization for monocrystalline silicon solar cells with different kinds of alkaline, Renew. Energy 29 (2004) 21012107.

[33] P.C. Searson, J.M. Macaulay, The fabrication of porous silicon structures, Nanotechnology 3 (4) (1992) 188191.

[34] Gunjan Aggarwal, Prabhash Mishra, Bipin Joshi, Harsh, S.S. Islam, Porous silicon surface stability: a comparative study of thermal oxidation techniques, J. Porous Mater. 21 (2014) 2329.

[35] Annett Thogersen, Josefine H. Selj, Erik S. Marstein, Oxidation effects on graded porous silicon anti-reflection coatings, J. Electrochem. 159 (5) (2012) D276 D281.

[36] C.H.F. Peden, J.W. Rogers Jr., N.D. Shinn, K.B. Kidd, K.L. Tsang, Thermally grown $\mathrm{Si}_{3} \mathrm{~N}_{4}$ thin films on Si (100) Surface and interfacial composition, Phys. Rev. B 47 (23) (1993) 15622.

[37] A. Thogersen, S. Diplas, J. Mayandi, T. Finstad, A. Olsen, J.F. Watts, M. Mitome, Y. Bando, An experimental study of charge distribution in crystalline and amorphous Si nanoclusters in thin silica films, J. Appl. Phys. 103 (2008) 024308.

[38] M. Terracciano, I. Rea, J. Politi, L. De Stefano, Optical characterization of aminosilane-modified silicon dioxide surface for biosensing, J. Eur. Opt. Soc. Rap. Publ. 8 (2013) 13075.

[39] L. Nony, T. Cohen-Bouhacina, J.P. Aimé, Dissipation induced by attractive interaction in dynamic force microscopy: contribution of adsorbed water layers, Surf. Sci. 499 (2002) 152160.

[40] A. Simon, T. Cohen-Bouhacina, M.C. Porté, J.P. Aimé, C. Baquey, Study of two grafting methods for obtaining a 3-Aminopropyltriethoxysilane monolayer on silica surface, J. Colloid Interface Sci. 251 (2002) 278283.

[41] Daniel D. Tune, Adam J. Blanch, Cameron J. Shearer, Katherine E. Moore, Moritz Pfohl, Joseph G. Shapter, Benjamin S. Flavel, Aligned carbon nanotube thin films from liquid crystal polyelectrolyte inks; chemically grafted carbon nanotube surface coverage gradients, ACS Appl. Mater. Interfaces 7 (2015) 2585725864

[42] Cameron J. Shearer, Kristina T. Constantopoulos, Nicolas H. Voelcker, Joseph G. Shapter, Amanda V. Ellis, Preparation and characterisation of vertically aligned single-walled carbon nanotube arrays on porous silicon, Smart Mater. V 7267 (2008) 111.

[43] Kristina T. Constantopoulos, Cameron J. Shearer, Amanda V. Ellis, Nicolas H. Voelcker, Joseph G. Shapter, Carbon nanotubes anchored to silicon for device fabrication, Adv. Mater. 22 (2010) 557571.

[44] Dong-Jie Guo, Shou-Jun Xiao, Bing Xia, Shuai- Wei, Jia Pei, Yi Pan, XiaoZeng You, Zhong-Ze Gu, Zuhong Lu, Reaction of porous silicon with both endfunctionalized organic compounds bearing $\alpha$-bromo and $\omega$-carboxy groups for immobilization of biomolecules, J. Phys. Chem. B 109 (43) (2005) 2062020628.

[45] Douglas B. Mawhinney, John A. Glass Jr., John T. Yates Jr., FTIR study of the oxidation of porous silicon, J. Phys. Chem. B 101 (7) (1997) 12021206.

[46] Martin J. Sweetman, Cameron J. Shearer, Joseph G. Shapter, Nicolas H. Voelcker, Dual silane surface functionalization for the selective attachment of human neuronal cells to porous silicon, Langmuir 27 (15) (2011) 94979503.

[47] Mahmood Torabi Angaji, Amir Zamani Zinali, Nader Taheri Qazvini, Study of physical, chemical and morphological alterations of smectite clay upon activation and functionalization via the acid treatment world, J. Nano Sci. Eng. 3 (2013) 161168.

[48] Barbara Stuart, Infrared spectroscopy: fundamentals and applications, Anal. Tech. Sci. (2004). ISBNs: 0-470-85427-8 (HB); 0-470-85428-6 (PB). 
[49] Michael P. Stewart, Jillian M. Buriak, Exciton-mediated hydrosilylation on photoluminescent nanocrystalline silicon, J. Am. Chem. Soc. 123 (32) (2001) 78217830.

[50] Yit-Lung Khung, Sean D. Graney, Nicolas H. Voelcker, Micropatterning of porous silicon films by direct laser writing, Biotechnol. Prog. 22 (5) (2006)
13881393

[51] Dan Rosu, Liliana Rosu, Constantin N. Cascaval, IR-change and yellowing of polyurethane as a result of UV irradiation, Polym. Degrad. Stab. 94 (4) (2009) 591596. 